\title{
Medidas Cefalométricas en Telerradiografías de Perfil de Pre-Escolares de 5 Años de la Ciudad de Temuco
}

\author{
Cephalometric Measurements in Lateral Radiographs of Five-Year-Old Pre-Schoolers in the City of Temuco
}

\author{
"Paulo Sandoval; ; Nerilda García; ${ }^{* * *}$ Antonio Sanhueza; ${ }^{* * * *}$ Andrea Romero \& ${ }^{* * * * *}$ Ricardo Reveco
}

\begin{abstract}
SANDOVAL, P.; GARCÍA, N.; SANHUEZA, A.; ROMERO, A. \& REVECO, R. Medidas cefalométricas en teleradiografías de perfil de pre-escolares de 5 años de la ciudad de Temuco. Int. J. Morphol., 29(4):1235-1240, 2011.

RESUMEN: La falta de parámetros cefalométricos validados a la población infantil de nuestra región para conocer el desarrollo craneofacial, hacen necesario tener mediciones que se aproximen a los diferentes patrones que presenta esta población. Debido a esto, surge el presente estudio de corte transversal de mediciones cefalométricas de niños de 5 años de edad, el cual se realizó con una muestra no probabilística consecutiva, constituida por 30 niños y 30 niñas de escuelas municipales de la ciudad de Temuco en el año 2009 con dentición temporal completa, no mapuches, con simetría facial, líneas medias centradas, con escalón mesial o plano post lácteo recto, sin tratamiento ortodóntico previo y con consentimiento de los padres por escrito. Se midieron 12 parámetros descritos por diferentes autores. Resultaron ser significativos estadísticamente los valores de eje facial y de altura facial posterior según el género. Respecto a los valores de Wits, los resultados muestran que los valores de la mediana para el género masculino es de 1,0 mm mientras que para el género femenino corresponde a un 1,5mm. Al aplicar el coeficiente de correlación de Pearson, para las variables estudiadas se encontró una relación lineal directamente proporcional entre los ángulos SNA y SNB $(0,75)$, SNA y BA-S-Na(0,5) y ángulo ANB y análisis de Wits $(0,6)$; mientras que al relacionar las variables ángulo SNB con ángulo Ba-S-Na(-0,7) la relación lineal es inversamente proporcional. Se concluye que existen diferencias a la norma internacional y se destaca en que a esta edad, los niños chilenos presentan una mandíbula levemente más retroposicionada.
\end{abstract}

PALABRAS CLAVE: Cefalometría; Niños; Preescolares.

\section{INTRODUCCIÓN}

El diagnóstico precoz de alteraciones craneofaciales y de la dentición es fundamental a la hora de prevenir, planificar y elaborar tratamientos que permitan interceptar o corregir anomalías dentomaxilares. Una de las herramientas auxiliares más utilizadas para lograr este objetivo es la cefalometría lateral, que es útil, tanto en la planificación del tratamiento ortodóntico como en la comparación de los resultados y en la valoración del crecimiento del paciente.

Diversos son los factores que deben ser evaluados a la hora de determinar un plan de tratamiento entre los que están: el género, la edad, la raza, el biotipo, el pronóstico del desarrollo y la severidad de la discrepancia dentomaxilar (Sinclair \& Little, 1985).

Las investigaciones en este ámbito han concluido que hay diferencias significativas entre diversos grupos étnicos y raciales (Velarde, 1974; Bishara \& Fernandez, 1985). Previo a estos estudios, se investigó diferencias étnicas de niños de origen caucásico y latinoamericano, midiendo 60 de estos últimos, cuyas edades oscilaron entre 4 y 5 años, con dentición, oclusión y proporciones faciales normales. Estos resultados los compararon con las medidas estándar para 5 años, obtenidas del estudio de crecimiento Iowa y concluyeron que el grupo latinoamericano exhibía mayores patrones de protrusión dental y esqueletal que los niños norteamericanos de origen caucásico (Bugg et al., 1973; Vann et al., 1978).

Sobreira en 1991, deduce que la oclusión normal de los dientes temporales permite el desarrollo normal de los dientes permanentes y de la cara, estableciendo que la presencia de maloclusión en la dentición temporal es un indicador de maloclusión en la dentición permanente, justifica

* Departamento de Odontología Integral, Universidad de La Frontera, Chile.

** Departamento de Odontología Integral, Universidad de La Frontera, Chile.

*** Departamento de Matemática y Estadística, Universidad de La Frontera, Chile.

***** Cirujano Dentista, Universidad de la Frontera, Chile.

Financiado por DIDUFRO 120438, Universidad de La Frontera, Chile. 
así la oportunidad del tratamiento temprano. Corrobora la existencia de dimorfismo sexual, debido a que los varones estudiados presentan mayores tasas de crecimiento de la longitud del maxilar y el cuerpo mandibular (Sobreira, 1991). Estas conclusiones se ven apoyadas por otros autores que afirman que si en la dentición primaria se hacen evidentes algunas maloclusiones, presentando además patrones craneofaciales distintivos, es necesario un diagnóstico precoz y un tratamiento ortodóntico individualizado, por lo que es importante utilizar variables cefalométricas sensibles durante este período del desarrollo (Tollaro et al., 1996).

Crecimiento y desarrollo craneofacial. Crecimiento se puede definir como el aumento de las dimensiones de la masa corporal. Esto es debido a la hipertrofia o hiperplasia de los tejidos constitutivos del organismo (Moyers, 1992), mientras que el desarrollo se refiere a los procesos de cambios cuanti-cualitativos que tienen lugar en el organismo y traen consigo el aumento en la complejidad de la organización e interacción de todos los sistemas. También se refiere a cambios unidireccionales que ocurren en un ser viviente desde constituirse como una simple célula hasta la muerte. Es necesario aclarar que las modificaciones en el tamaño y en la función de un órgano no pueden ser separadas. El crecimiento y desarrollo representan una diversidad y continuidad de interacciones entre la herencia y el ambiente, manteniendo las proporciones (Moyers).

Normalidad en dentición temporal. La dentición decidua es la primera dentición del ser humano, consta de 20 dientes que inician su erupción aproximadamente a los 6 meses de edad con los incisivos centrales inferiores y termina aproximadamente entre los 2 años 6 meses y los 3 años de edad con la erupción de los segundos molares temporales superiores. Concluida la erupción de los dientes deciduos, continúan algunos cambios en la medida de los arcos en los tres planos del espacio debido al crecimiento y desarrollo de los maxilares y de las estructuras vecinas. Dichas medidas pueden ser útiles para determinar la normalidad de los cambios que ocurren en esta etapa y lo que sucederá en la dentición decidua y permanente (Van der Linden, 1986). La mayoría de los arcos primarios son ovoides y muestran menos variabilidad en su formación que los permanentes (Moyers).

Entre los signos de normalidad en dentición temporal son: piezas anteriores separadas, presencia de espacios primates, leve sobre mordida y resalte, plano terminal recto, relación canina o signo canino normal, inclinación casi vertical de dientes anteriores, forma del arco ovoide, línea media coincidente, relación radial posterior que corresponde a la relación de los molares en el plano horizontal con los superiores más vestibular que los inferiores y plano oclusal, aproximadamente horizontal, sin curva (Escobar, 2004; Moyers).
La determinación de la armonía de las líneas faciales y un perfecto equilibrio entre sus partes, incluyendo obviamente los dientes, son imprescindibles para la comprensión y determinación de la normalidad.

Análisis cefalométrico. La cefalometría como instrumento de diagnóstico, fue descrita por B. H. Broadbent en la primera mitad del siglo XX (Broadbent, 1931), los primeros reportes de su uso clínico los dieron Brodie, Down y Goldstein (Brodie et al., 1938). En la década de los 50, se señaló que muchos de los problemas, fracasos y recidivas en tratamientos de ortodoncia, tenían por origen la falta de un análisis cefalométrico completo de las estructuras faciales y óseas (Goldstein, 1953; Koski, 1953; Richardson, 1954; Herzberg, 1954). Entre los análisis cefalométricos, se encuentra el análisis de Downs, el análisis de Steiner, el análisis de Bjork y auxiliares de diagnóstico, como el análisis de Tweed y el análisis de Wits.

También ha sido utilizado para el estudio longitudinal de niños, para evaluar los cambios propios del crecimiento. Su aplicación frecuente en grupos importantes de la población permite disponer de medidas de tendencia central, estadígrafos con los cuales pueden compararse los individuos (Escobar). El cefalograma lateral es entonces, un aspecto bidimensional de una estructura tridimensional y de base para una descripción morfológica de la cara y la dentición, así como para la identificación de las anomalías dentales y esqueletales (Águila, 1996).

La descripción cefalométrica ayuda en la especificación, localización y comprensión de las anormalidades. Esta descripción comprende tres tipos de comparaciones: comparación con una norma, comparación con un ideal o comparación con un sujeto. Existen en la actualidad numerosos métodos de análisis cefalométricos, pero debido a que este estudio tiene solamente un objetivo diagnóstico, se tomaran en cuenta las siguientes mediciones (Fig. 1).

\section{Análisis de Steiner}

Ángulo SNA (Análisis del Maxilar Superior): Ángulo formado por el plano S-N y N- Punto A. El valor normal es de $82^{\circ}+2^{\circ}$. Determina una medida angular que muestra la posición anteroposterior de la maxila con relación a la base del cráneo.

Ángulo $S N B$ (Análisis de la mandíbula): Ángulo formado por el plano S-N y N-Punto B. El valor normal es de $80^{\circ}+$ 2. Indica la relación anteroposterior de la mandíbula con relación al cráneo. La medida angular muestra si la mandíbula está prognata, retrognata o bien posicionada con relación a la base craneana. 
Ángulo ANB (Análisis de la Relación Intermaxilar): Corresponde al ángulo formado por la unión de los Puntos A, $\mathrm{N}$ y B. El valor normal es de $2^{\circ}+2^{\circ}$. Indica la relación que tienen las bases apicales entre sí, permitiéndonos determinar la clase esqueletal de Angle. Un ángulo mayor de $2^{\circ}$ indica tendencia a clase II y un ángulo menor de $2^{\circ}$, tendencia a clase III. Por norma debiera medir $2^{\circ}$ (Gregoret, 1997).

\section{Análisis de Ricketts}

Eje Facial: Describe la dirección del crecimiento del mentón y forma un ángulo recto con el plano Basion- Nasion y su DS es de $3^{\circ}$ (Gregoret).

\section{Análisis de Björk- Jaraback}

Ángulo goníaco: Está formado por las tangentes al borde posterior de la rama ascendente de la mandíbula (Ar-Go) $\mathrm{y}$ al borde inferior al cuerpo de la misma (Go-Me). La norma según Björk es de $130^{\circ}$.

Ángulo Ba-S-N. Es la unión de la base craneal anterior con la posterior y es el ángulo formado por las líneas trazadas desde nasion al punto silla y desde este al punto articular; su medida es $122^{\circ}$.

Relación entre altura facial posterior y altura facial anterior: que indica dirección del crecimiento rotacional craneofacial y es la resultante de la división entre altura facial posterior y altura facial anterior multiplicado por 100, cuya norma es: braquifacial: 64 a $80 \%$; mesofacial:59 a $63 \%$ y dólicofacial: 54 a 58\% (Gregoret).

\section{Análisis de Wits}

Se proyectan los puntos A y B perpendicularmente al plano oclusal y se mide la distancia entre ambos puntos. El valor normal es de $0 \mathrm{~mm}$ en mujeres y de () $1 \mathrm{~mm}$ en varones, donde el punto A estaría $1 \mathrm{~mm}$ por detrás del punto B. En términos generales, en la clase I, la proyección del punto A y B generalmente coinciden. En las clases II, la proyección del punto B estaría delante del punto A dando un valor de signo positivo; en las clases III, la proyección del punto B se encontraría delante del A dando un valor de signo negativo (Jacobson, 1975).

Respecto a la telerradiografía lateral, se considerará aceptable aquella radiografía de perfil en la que el paciente este en oclusión céntrica, un adecuado perfil blando con los labios en reposo, que no tenga dobles contornos o estos sean mínimos y se encuentre estandarizada.

\section{MATERIAL Y MÉTODO}

Estudio de corte transversal, cuyo universo son los niños de la ciudad de Temuco de 5 años de edad, que asisten a jardines infantiles municipales. Se seleccionó una muestra de 60 preescolares (50\% niños y $50 \%$ niñas) con dentición temporal completa, no mapuches, con simetría facial, líneas medias centradas, con escalón mesial o plano post lácteo recto. Se excluyeron de la muestra niños que recibieron algún tratamiento ortodóntico. Los padres o apoderados realizaron consentimiento informado escrito para autorizar la participación en el estudio. El período de reclutamiento fue el segundo semestre del año 2008.

La toma de radiografías se realizó en la Clínica Docente Asistencial (CODA), perteneciente a la Universidad de la Frontera. Se utilizó el equipo Bluex Imagen STI Panceph Pantos 16 (2004), siendo estas realizadas por el mismo operador, usando la misma técnica en cada una de ellas. Las mediciones cefalométricas fueron realizadas por un operador distinto, sin conocimiento del estudio (Fig. 1).

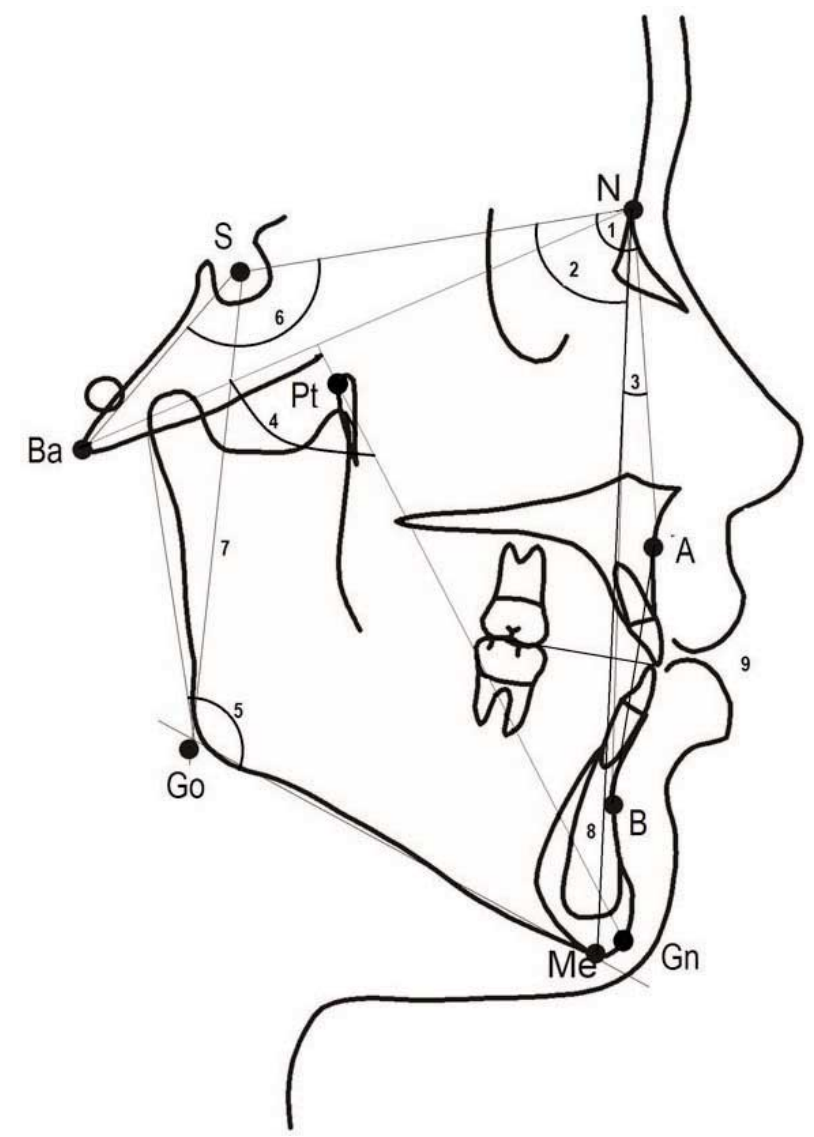

Fig. 1. Medidas cefalométricas tomadas de los análisis de Steiner, Rickets, Jarabak y Wits Apraisal. 1. Ángulo SNA; 2. Ángulo SNB; 3. Ángulo ANB; 4. Eje facial; 5. Ángulo goníaco; 6. Ángulo de la Silla; 7. Altura facial posterior; 8. Altura facial anterior; 9.Wits. 
La magnificación del equipo fue considerada y correspondió a $11 \%$.

Para el análisis estadístico de los datos obtenidos se hizo uso de medidas descriptivas tradicionales, tales como promedio, mediana, desviación estándar y correlación. Además, se utilizó métodos inferenciales incluyendo la prueba de $t$ de Student para muestras independientes.

\section{RESULTADOS}

Los promedios con las respectivas desviaciones estándar de las medidas cefalométricas y su asociación con genero, obtenida a través de la prueba t de Student, son descritos en Tabla I. Basados en este análisis, se encontró que las variables Eje Facial y Altura Facial Posterior están asociadas con la variable Género (valor-p $\leq 0.05$ ). Desde la Tabla I, para la variable Wits, los resultados muestran que el pro- medio es afectado por valores extremos, por lo que es necesario reportar la mediana, que para el sexo masculino es de $1,0 \mathrm{~mm}$ mientras que para el sexo femenino corresponde a un $1,5 \mathrm{~mm}$. La Tabla II muestra que el $25,4 \%$ de los individuos es clase I, el $57,6 \%$ es clase II y el $17 \%$ es clase III, según el análisis de Wits.

Respecto al crecimiento rotacional, se encontró que los niños presentaron un promedio de 65,58\% (DS 6,32\%) de crecimiento y las niñas un $62,57 \%$ (DS 5,36\%); Tabla III.

Al aplicar el coeficiente de correlación de Pearson para las variables estudiadas, se encontró una relación lineal directamente proporcional entre las variables Angulo SNA y Angulo SNB ( $\mathrm{r}=0,75)$, Angulo SNA y Angulo BA$\mathrm{S}-\mathrm{N}(\mathrm{r}=0,5)$, y Angulo ANB y análisis de Wits $(\mathrm{r}=0,6)$; mientras que esta relación lineal es inversamente proporcional $(\mathrm{r}=-0,7)$ para las variables Angulo SNB con Angulo Ba-SN.

Tabla I. Descripción de las medidas cefalométricas según sexo.

\begin{tabular}{lccccc}
\hline \multirow{2}{*}{ Medidas Cefalométricas } & \multicolumn{4}{c}{ Sexo } & Valor p \\
\cline { 2 - 5 } & \multicolumn{2}{c}{ Masculino } & \multicolumn{2}{c}{ Femenino } & \\
\cline { 2 - 5 } & Promedio & DS & Promedio & DS \\
\hline SNA $\left(^{\circ}\right)$ & 85,2 & 4,2 & 84,2 & 3,9 & 0,3359 \\
SNB & 78,77 & 4,2 & 77,4 & 3,4 & 0,2309 \\
ANB & 6,6 & 1,8 & 7 & 2,4 & 0,4953 \\
EJE FACIAL & 92,8 & 4,7 & 96,2 & 4,9 & 0,0080 \\
ÁNGULO GONIACO & 129,7 & 5,6 & 128,6 & 5,4 & 0,4579 \\
Ba-S-Na & 128,2 & 5,2 & 129,8 & 4,9 & 0,2304 \\
AFA & 100,8 & 7 & 102,3 & 5,6 & 0,3745 \\
AFP & 65,8 & 3,6 & 63,8 & 4,2 & 0,05 \\
WITS & 1,1 & 1,9 & 1,1 & 2,4 & 0,9952 \\
\hline
\end{tabular}

Tabla II. Distribución del análisis de WITS según la clase esqueletal.

\begin{tabular}{lcc}
\hline Clase esqueletal & Número & Porcentaje \\
\hline Clase I & 15 & $25,4 \%$ \\
Clase II & 34 & $57,6 \%$ \\
Clase III & 10 & $17,0 \%$ \\
\hline
\end{tabular}

Tabla III. Promedio del crecimiento rotacional según sexo.

\begin{tabular}{lcc}
\hline Sexo & Promedio & Desviación Estándar \\
\hline Masculino & 65,58 & 6,32 \\
Femenino & 62,57 & 5,96 \\
Prome dio & 64,07 & 6,00 \\
\hline
\end{tabular}

\section{DISCUSIÓN}

En Chile no existen estudios en donde se determine los valores de normalidad para el rango de edad de este estudio. Sin embargo, existen en la literatura ciertos estudios que permiten relacionar sus resultados con los nuestros.

Rocha \& Viera de Siqueira (2006), en un estudio cefalométrico de niños de la ciudad de Belo horizonte, Brasil, establecieron que el promedio de la variable Angulo SNA en niños de 5 años fue de $82,1^{\circ}$. Otro estudio en pacientes del mismo grupo etario, llevado a cabo en la ciudad de México coinciden en que la medida de este ángulo reportada para 
género masculino fue de $82,27^{\circ}$ (DS $3,29^{\circ}$ ) y para género femenino de $82,45^{\circ}\left(\mathrm{DS} 3,29^{\circ}\right)$ (Flores et al.), siendo la media resultante de este estudio ligeramente mayor para género masculino de $85,2^{\circ}$ (DS $4,2^{\circ}$ ) y para género femenino de $84,2^{\circ}$ $\left(\mathrm{DS} 3,9^{\circ}\right)$. En individuos coreanos se encontró un valor promedio de $84^{\circ}$, pero con una DS $2,75^{\circ}$, que indica un amplio rango de posición de la maxila (Choi et al., 2010). Estos valores son mayores a los reportados por Tollaro et al. en niños y niñas de Italia, $79,8^{\circ}$ (DS 2,91 ${ }^{\circ}$ ).

Respecto al el valor de ángulo SNB (posición de la mandíbula con respecto a la base de cráneo), mostró que para el género masculino la media fue de $78,77^{\circ}\left(\mathrm{DS} 4,2^{\circ}\right.$ ) y para el género femenino de $77,4^{\circ}$ (DS3, $4^{\circ}$ ), siendo similares al estudio mexicano cuyo reporte muestra una diferencia entre masculino $\left(77,25^{\circ}\right.$ DS $\left.3,39^{\circ}\right)$ y femenino $\left(77,19^{\circ}\right.$ DS2.09 $)$ (Flores et al., 2004). Así, este estudio arrojó valores menores que el reportados por Rocha \& Viera de Siqueira en cual fue $78,03^{\circ}$ para género masculino. Choi et al., reportaron un valor promedio de $77,72^{\circ}$ pero con una DS $2,76^{\circ}$ que indica un amplio rango de posición de la mandíbula. Estos valores son mayores al igual que en el SNA a los reportados por Tollaro et al. en niños y niñas de Italia, $76,35^{\circ}\left(\mathrm{DS} 2,85^{\circ}\right)$.

En cuanto a la relación antero posterior entre las bases óseas, dada por el ángulo ANB, comparado con los estudios antes mencionados, en nuestra muestra se encontró una mandíbula más retroposicionada en relación a la maxila. Esto se comprueba con lo encontrado por Tanabe et al. (2002), por lo que podemos inferir de nuestros resultados que los preescolares chilenos presentan una mandíbula levemente mas retroposicionada que las otras etnias. Contrariamente en individuos koreanos se encontró un valor promedio de 5,28 pero con una DS 1,91 que indica un amplio rango de posición de la maxila (Choi et al.).

Respecto al ángulo goníaco, en un estudio realizado en niños peruanos de 5 a 9 años se reportó un promedio para esta medida cefalométrica de $123,38^{\circ}$ (DS4,5 $)$ (Padilla et al., 2009), siendo esta medida menor a la arrojada en este estudio, que para género masculino fue de $129,7^{\circ}\left(\mathrm{DS} 5,6^{\circ}\right)$ y $128,6^{\circ}$ (DS 5,4) para género femenino.

En cuanto a la altura facial anterior y posterior el estudio de niños peruanos reportaron una media de $112,67 \mathrm{~mm}$ (DS 6,9mm) y $67,3 \mathrm{~mm}$ (DS 6,6mm) respectivamente para niños menores de 9 años (Padilla et al.), resultados que se diferencian de los de nuestro estudio en que en el género masculino se registró $100,8 \mathrm{~mm}$ (DS $7 \mathrm{~mm}$ ) y en el femenino $102,3 \mathrm{~mm}$ (DS 5,6 mm) para la altura facial anterior y $65,8 \mathrm{~mm}$ (DS 3,6mm) para los varones y $63,8 \mathrm{~mm}$ (DS4,2mm) para las damas en la altura facial posterior. En el estudio de niños koreanos se muestra un promedio de crecimiento rotacional de 62, 47mm DS3,57 levemente inferior a los 64,07 DS6,0 de nuestro estudio.

En relación a la base craneal Tollaro et al. reportan un ángulo de $131,56^{\circ}$ (DS $\left.4,44^{\circ}\right)$, en cambio en nuestro estudio encontramos en promedio $128,2^{\circ}$ para niños y $129,8^{\circ}$ en niñas, lo que se considera dentro de rangos similares.

Las media del análisis de Wits resultó en 1,1mm (DS $2 \mathrm{~mm}$ ), muy diferente a lo reportado por Nogueira \& Oliveira (2003), el cual fue de $-0,08 \mathrm{~mm}$ (DS $1,83 \mathrm{~mm}$ ), medida que se invalida por lo amplio de su desviación estándar, debe reportarse la mediana, medida no encontrada en otros estudios. Choi et al. reporta una norma coreana de -0,84 (DS 1,91) que indica una característica orientada a clase III en países asiáticos distinta a nuestros resultados más cercanos a clase I.

Se concluye que existen diferencias a la norma internacional. Entre 5 y 6 años de edad, los niños chilenos presentan una mandíbula levemente más retroposicionada, aunque de acuerdo a la relación maxilomandibular respecto al plano oclusal un quinto de los individuos son clase III, lo que una característica de prognasia mandibular.

SANDOVAL, P.; GARCÍA, N.; SANHUEZA, A.; ROMERO, A. \& REVECO, R. Cepahlometrics measurements in lateral radiographs of five-year-old preschoolers in the city of Temuco . Int. J. Morphol., 29(4):1235-1240, 2011.

SUMMARY: The lack of validated cephalometric parameters in the pediatric population of our region to learn craniofacial development, there is a need to know approximate measurements of different patterns that present in the child population. The present study of cross-sectional cephalometric measurements in five-year-old children, which was performed with a straight non-probability sample, consisting of 30 boys and 30 girls from municipal schools in Temuco in 2009 with full dentition, non-Mapuche with facial symmetry, centered midline, with tray or flat mesial post straight, without prior orthodontic treatment with parental consent in writing. 12 parameters were measured as described by different authors. According to the Student's tests statistically significant facial axis values and posterior facial height were found according to sex. With respect to the values of Wits, the results show that the median values for male is $1.0 \mathrm{~mm}$ while for the female corresponds to $1.5 \mathrm{~mm}$. By applying the Pearson correlation coefficient for the variables studied we found direct proportional linear relationship between SNA and SNB angles (0.75), SNA and BA-S-Na (0.5) and ANB angle and analysis Wits (0.6), while relating the angle variables SNB angle Ba-S-Na (-0.7) the linear relationship is inversely proportional. We conclude that there are differences to the international standard and noted that at this age Chilean children have a jaw slightly in retroposition. 


\section{REFERENCIAS BIBLIOGRÁFICAS}

Águila, J. Manual de cefalometría. Barcelona, Actualidades Médico Odontológicas, 1998.

Bishara, S. E. \& Fernandez, A. G. Cephalometric comparisons of the dentofacial relationships of two adolescent populations from Iowa and Northern Mexico. Am. J. Orthod., 88(4):31422, 1985.

Broadbent, B. H. A new X-Ray technique and its application to Orthodontics. Angle Orthod., 1(2):45-66, 1931.

Brodie, A.; Downs, W.; Goldstein, A \& Myer, E. Cephalometric appraisal of orthodontic results. Angle Orthod., 8(4):261-5, 1938.

Bugg, J. L. Jr.; Canavati, P. S. \& Jennings, R. E. A cephalometric study for preschool children. ASDC J. Dent. Child., 40(2):1034, 1973.

Choi, H. J.; Kim, J. Y.; Yoo, S. E.; Kwon, J. H. \& Park, K. Cephalometric characteristics of Korean children with Class III malocclusion in the deciduous dentition. Angle Orthod., 80(1):86-90, 2010.

Escobar, F. Odontología Pediátrica. $2^{\circ}$ ed. Caracas, Amolca, 2004.

Flores, L.; Fernadez, M. \& Heredia, E. Valores cefalometricos en niños preescolares del jardín de niños CENDI UNAM. Rev. Odontol. Mex., 8(1-2):17-23, 2004.

Goldstein, A. The dominance of the morphological pattern: implications for treatment. Angle Orthod., 23(4):187-95, 1953.

Gregoret, J. Ortodoncia y cirugía ortognática, Diagnóstico y planificación de tratamiento. Barcelona, Publicaciones Médicas, 1997.

Herzberg, B. The Tweed formula anchorage preparation and facial esthetics. Angle Orthod., 24(3):170-7, 1954.

Jacobson, A. The "Wits" appraisal of jaw disharmony. Am. J. Orthod., 67(2):125-38, 1975.

Koski, K. Analysis of profile roentgenograms by means of a newcircle method. Dent. Rec., 73:704-13, 1953.

Moyers, R. Manual de Ortodoncia. $4^{\mathrm{a}}$ ed., Buenos Aires, Panamericana, 1992.

Nogueira, T. G. \& Oliveira, A. G. Determinação do padrão cefalométrico de brasileiros, da raça branca com oclusão normal na fase da dentadura decídua. J. Bras. Ortodon. Ortop. Facial, 8(46):274-92, 2003.

Padilla, C. T. C.; Silva-Esteves, R. J. F. \& Morzán, V. E. Características esqueléticas evaluadas por los análisis cefalométricas de Jaraback, McNamara y DiPaolo en niños peruanos de 5 a 12 años de edad. Odontol. Pediatr., 8(2):17-24, 2009.

Richardson, J. Roentgenographic evaluation of orthodontic treatment. Angle Orthod., 24(1):31-7, 1954.

Rocha M. \& Viera de Siqueira, V. Estudo cefalométrico das características ântero-posteriores em jovens com dentadura decídua. Rev. Dent. Press Ortodon. Ortop. Facial, 11(5):93-103, 2006.

Sinclair, P. \& Little, R. Dentofacial maturation of untreated normals. Am. J. Orthod. Dentofacial Orthop., 88(2):146-56, 1985

Sobreira, J. Dentadura decídua: estudo cefalométrico de estruturas craniofaciais em indivíduos brasileiros dotados de oclusão dentária normal. Dissertação (Mestrado em Ortodontia). Faculdade de Odontologia de Piracicaba, Universidade Estadual de Campinas, Piracicaba, 1991.

Tanabe, Y.; Taguchi, Y. \& Noda, T. Relationship between cranial base structure and maxillofacial components in children aged 3-5 years. Eur. J. Orthod., 24(2):175-81, 2002.

Tollaro, I.; Bacetti, T. \& Franchi, L. Floating norms for the assessment of craniofacial pattern in the deciduos dentition. Eur. J. Orthod., 18(4):359-65, 1996.

Van der Linden, F. Desenvolvimento da dentição. São Paulo, Quintessence Editora, 1986.

Vann, W. F. Jr.; Dilley, G. J. \& Nelson, R. M. A cephalometric analysis for the child in the primary dentition. ASDC J. Dent. Child., 45(1):45-52, 1978.

Velarde, E. A. Cephalometric norms for the Mexican population using the Ricketts, Steiner, and Tweed analyses. Master's thesis, Loma Linda University Graduate School, 1974.

Dirección para correspondencia:

Paulo Sandoval

Departamento de Odontología Integral

Universidad de la Frontera

Temuco

CHILE

Email: nery.ga@gmail.com

Recibido : 05-07-2011

Aceptado: 12-09-2011 